\title{
MODELING OF ACOUSTIC FIELDS GENERATED BY FLOW PAST AN OPEN CAVITY*
}

\author{
H.T.Banks ${ }^{\dagger}$ D. Rubio ${ }^{\ddagger}$ and R. Smith ${ }^{\S}$ \\ Center for Research in Scientific Computation \\ North Carolina State University \\ Raleigh, NC 27695
}

\begin{abstract}
Fluid flow, passing at high speed over an open cavity on a surface, interacts with the structure producing pressure fluctuations within the cavity. If the intensity of the acoustic pressure is large, it may damage stored instrumentation and electronic devices and induce structural vibration and fatigue. Several experimental and numerical investigations have been conducted with the goal of control the acoustic oscillations. However, the full capability of these methods have not yet been realized due to lack of an appropriate model to use in control design. Here we present one such mathematical model for the acoustic field in an open cavity in a plane where flow/acoustic interactions and nonlinear effects are considered.
\end{abstract}

\section{INTRODUCTION}

When fluid at high speed flows over an open cavity, large acoustic pressure fields inside the cavity are produced by fluid/structure interactions at the downstream end of the cavity. In the case of an airplane, acoustic waves are created in wheelwells during takeoff and landing, and in weapon and/or surveillance bays during flight. Pressure fluctuations can be so high as to damage stored instrumentation

\footnotetext{
* Research supported in part by the U.S. Air Force Office of Scientific Research under grant AFOSR 49620-98-1-0180. Copyright (C)1999 by the authors. Published by the American Institute of Aeronautics and Astronautics, Inc. with permission.

$\dagger^{\dagger}$ University Professor and Drexel Professor of Mathematics

$\ddagger$ Visiting Assistant Professor of Mathematics

$\S$ Associate Professor of Mathematics
}

or structures (see for instance [6]). This increases the importance of attenuating the pressure field created within the cavity. In order to succeed in systematic noise reduction, a mathematical model that captures the essential features of the physical process will be fundamental.

A number of authors have studied the physical mechanism that induces the acoustic waves along with different means to control it. These efforts have involved a variety of cavity shapes and Mach numbers. It is now known that this physical mechanism results in different characteristics depending on the ratio length/depth $(l / d)$ of the cavity. In this regard, a cavity is usually classified as shallow or deep depending on whether its ratio $l / d$ is greater or is less than 2 , respectively. In our considerations here we discuss flow over a shallow cavity.

In [6], H.H.Heller and D.B.Bliss consider a shallow cavity for analysis and experiments. They describe the fluid/cavity interaction as a six step feedback loop where instabilities of the shear layer cause a mass addition/removal process at the cavity downstream end. They predict the mode shapes and amplitudes and implement suppression techniques to reduce them. In [3], Cain, Bower, McCotter and Romer consider the cases of supersonic and subsonic/transonic flow and give a classification of the flow type along with the corresponding pressure profile for each case. They focus on the case of an "open cavity flow" consisting of cavities satisfying the condition $l / d<10$, and describe the problem as a fourstep process where each step is modeled and used to develop a code for simulation. In [8], Rockwell and Nausdacher consider three different groups based on 
what originates and sustains the oscillations in the cavity. They discuss the physics characteristics and mathematical models for the frequencies and amplitudes in each case. In their paper they present an extensive summary and comparison of efforts prior to 1978 in terms of analysis, attenuation means and experimental results.

Here we present a physical-based nonlinear mathematical model for the fluid/structure interaction in a two-dimensional shallow cavity with $2<$ $l / d<10$. In this case the fluid-induced oscillation process begins when a boundary layer separates at the upstream end of the cavity, creating an unstable wave. This wave propagates and amplifies downstream across the top of the cavity until it reaches the downstream end of the cavity, where it interacts with the structure, generating an acoustic field. The acoustic wave then propagates back upstream inside the cavity until it reaches the upstream end, feeding the disturbances in the shear layer (see, e.g., [3], $[6],[8])$. In most of the efforts to date, the authors consider a linear model along with a semi-empirical formula that predicts modal frequencies (see [3], [6], [8], [9]).

We specify the characteristics of the flow considering two large regions, one above the shear layer and one beneath it. The governing equations are derived where in each case the state equation for perfect gas is considered. Boundary conditions and shear layer interface conditions are defined. A weak formulation suitable for finite element computations is then given.

\section{MATHEMATICAL MODEL}

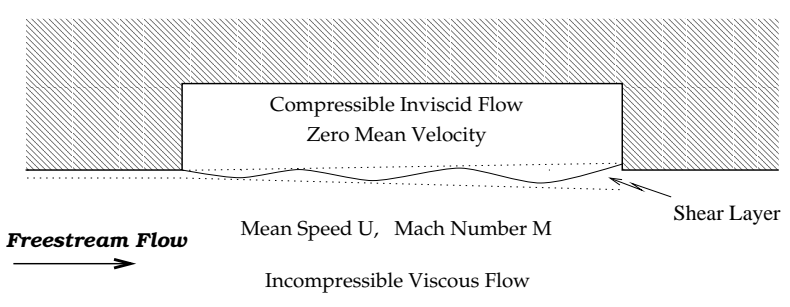

Figure 1: Two-Dimensional Open Cavity under High-Speed Flow

The model presented here is derived from the conservation laws of mass and momentum and the state equation, which relates the pressure, the density and the temperature of the fluid.
We consider the two dimensional problem for a rectangular shallow cavity where the ratio length/depth is greater than 2 but less than 10 (referred to as "open cavity flow" by Cain et al.), as depicted in Figure 1. The freestream flow is assumed to have a uniform mean speed denoted by $U$ with a Mach number $M$. The fluid in this region is assumed to be a perfect gas, with small variations in density, and thus it may be regarded as incompressible and viscous. However, inside the cavity, it is assumed that the viscous effects above the shear layer do not play a key role in the creation and propagation of the acoustic waves. Hence, as our first approximating model we assume a compressible inviscid fluid above the shear layer, inside the cavity, and incompressible viscous flow everywhere else.

We also assume that the fluid temperature is the same outside and inside the cavity, and thus the stationary values for pressure and density are the same inside and outside of the cavity.

Based on these considerations, we proceed to derive the equations for the motion of the flow.

\section{Governing Equations for the Freestream Flow}

Consider first the freestream flow, which is assumed to be viscous, incompressible with a uniform mean speed $U$ (see Figure 1). Therefore, its motion may be described by the Navier-Stokes equations

$$
\begin{aligned}
\frac{\partial \mathbf{u}_{f}}{\partial t}+\left(\mathbf{u}_{f} \cdot \nabla\right) \mathbf{u}_{f}= & \frac{1}{\rho_{f}} \nabla \cdot\left[\mu\left(\nabla \mathbf{u}_{f}+\left(\nabla \mathbf{u}_{f}\right)^{T}\right)\right] \\
& -\frac{\nabla p_{f}}{\rho_{f}}
\end{aligned}
$$

the continuity equation or conservation of mass law,

$$
\frac{\partial \rho_{f}}{\partial t}=-\nabla \cdot\left(\rho_{f} \mathbf{u}_{f}\right)=-\nabla \rho_{f} \cdot \mathbf{u}_{f}
$$

and the state equation for perfect gas

$$
p_{f}=\rho_{f} R T,
$$

where $\mathbf{u}_{f}=\left(u_{f}, v_{f}\right)$ is the velocity of the flow, $\rho_{f}$ is density, $\mu$ is the viscosity coefficient, $p_{f}$ is the pressure, $R$ is a constant, called the gas constant, and $T$ is the temperature. If the fluid's viscosity $\mu$ is taken to be constant in space, the equation of motion (1) becomes

$$
\frac{\partial \mathbf{u}_{f}}{\partial t}+\left(\mathbf{u}_{f} \cdot \nabla\right) \mathbf{u}_{f}=\frac{\mu}{\rho_{f}} \Delta \mathbf{u}_{f}-\frac{\nabla p_{f}}{\rho_{f}} .
$$


Remark 1 If $\mu$ does not depend on space we have

$$
\nabla \cdot\left[\mu\left(\nabla \mathbf{u}_{f}+\left(\nabla \mathbf{u}_{f}\right)^{T}\right)\right]=\mu \nabla \cdot\left(\nabla \mathbf{u}_{f}+\left(\nabla \mathbf{u}_{f}\right)^{T}\right)
$$

and since

$$
\nabla \cdot\left(\nabla \mathbf{u}_{f}\right)^{T}=\nabla\left(\nabla \cdot \mathbf{u}_{f}\right),
$$

which vanishes for incompressible flow, it turns out that

$$
\nabla \cdot\left[\mu\left(\nabla \mathbf{u}_{f}+\left(\nabla \mathbf{u}_{f}\right)^{T}\right)\right]=\mu \nabla \cdot \nabla \mathbf{u}_{f}=\mu \Delta \mathbf{u}_{f} .
$$

The speed of sound of a perfect gas, denoted by $c$, is given by

$$
c^{2}=\gamma R T, \quad \gamma=\frac{C_{p}}{C_{v}},
$$

where $C_{p}$ is the specific heat at constant pressure and $C_{v}$ is the specific heat at constant volume. In particular, $\gamma=1.4$ for air. Then, the equation of state is given by

$$
p_{f}=\frac{c^{2}}{\gamma} \rho_{f}
$$

Note that we assume that the temperature is constant, thus the speed of sound $c$ is also constant. By defining

$$
c_{0}^{2}=\frac{c^{2}}{\gamma}
$$

we may write

$$
p_{f}=c_{0}^{2} \rho_{f} .
$$

Combining the continuity equation (2) and the state equation (6) we have

$$
\frac{\partial p_{f}}{\partial t}=-\nabla \cdot\left(p_{f} \mathbf{u}_{f}\right)=-\nabla p_{f} \cdot \mathbf{u}_{f}
$$

Since variations in fluid density in the freestream flow are very small, we may approximate $\rho_{f}$ by its mean value $\rho_{0}$. Therefore, the equations of motion for the freestream flow reduced to

$$
\begin{aligned}
\frac{\partial \mathbf{u}_{f}}{\partial t}+\left(\mathbf{u}_{f} \cdot \nabla\right) \mathbf{u}_{f} & =\frac{\mu}{\rho_{0}} \Delta \mathbf{u}_{f}-\frac{\nabla p_{f}}{\rho_{0}} \\
\frac{\partial p_{f}}{\partial t} & =-\nabla \cdot\left(p_{f} \mathbf{u}_{f}\right) \\
p_{f} & =c_{0}^{2} \rho_{f} .
\end{aligned}
$$

Remark 2 Note that we approximate $\rho_{f}$ by $\rho_{0}$ only in the Navier-Stokes equations.

\section{Governing Equations for the Acoustic Waves}

The acoustic waves inside the cavity propagate in a region determined by the shear layer and the cavity. It was observed in experiments conducted by Heller and Bliss, [6], that the cavity fluid can be described by the acoustic wave equation; hence the fluid may be regarded as compressible and inviscid as depicted in Figure 1. In order to model the motion of the acoustic waves we consider the basic equations of balance of mass and momentum and the equation of state.

Hence, the equations that describe the fluid motion are the Euler's equations

$$
\frac{\partial \mathbf{u}_{c}}{\partial t}+\left(\mathbf{u}_{c} \cdot \nabla\right) \mathbf{u}_{c}=-\frac{\nabla p_{c}}{\rho_{c}}
$$

the continuity equation

$$
\frac{\partial \rho_{c}}{\partial t}=-\nabla \cdot\left(\rho_{c} \mathbf{u}_{c}\right)
$$

and the state equation

$$
p_{c}=c_{0}^{2} \rho_{c} .
$$

Here $\mathbf{u}_{c}=\left(u_{c}, v_{c}\right), \rho_{c}$ and $p_{c}$ are the velocity, density and pressure of the fluid in the cavity and above the shear layer, respectively.

Remark 3 Note that the state equation is analogous to that for the fluid below the shear layer (10).

We now apply the time derivative to Euler's equation (11)

$$
\begin{aligned}
\frac{\partial^{2} \mathbf{u}_{c}}{\partial t^{2}}+\frac{\partial}{\partial t} & {\left[\left(\mathbf{u}_{c} \cdot \nabla\right) \mathbf{u}_{c}\right]=-\frac{\partial}{\partial t}\left(\frac{\nabla p_{c}}{\rho_{c}}\right) } \\
& =\frac{1}{\rho_{c}^{2}} \frac{\partial \rho_{c}}{\partial t} \nabla p_{c}-\frac{1}{\rho_{c}} \nabla\left(\frac{\partial p_{c}}{\partial t}\right) .
\end{aligned}
$$

The above equation, the continuity equation (12) and the approximated state equation (13) yield

$$
\begin{aligned}
\frac{\partial^{2} \mathbf{u}_{c}}{\partial t^{2}}+\frac{\partial}{\partial t}\left[\left(\mathbf{u}_{c} \cdot \nabla\right) \mathbf{u}_{c}\right] & \\
= & -\frac{\nabla \rho_{c}}{\rho_{c}} \cdot \mathbf{u}_{c} \frac{\nabla p_{c}}{\rho_{c}}-\nabla \cdot \mathbf{u}_{c} \frac{\nabla p_{c}}{\rho_{c}} \\
& +c_{0}^{2} \frac{\nabla\left[\nabla \cdot\left(\rho_{c} \mathbf{u}_{c}\right)\right]}{\rho_{c}} .
\end{aligned}
$$


By means of the following identities (see [4])

$$
\begin{aligned}
& \Delta \mathbf{u}=\nabla(\nabla \cdot \mathbf{u})-\nabla \times(\nabla \times \mathbf{u}), \\
& \nabla \times(f \mathbf{u})=f \nabla \times \mathbf{u}+\nabla f \times \mathbf{u}
\end{aligned}
$$

and

$\nabla \times(\mathbf{u} \times \mathbf{w})=\mathbf{u} \nabla \cdot \mathbf{w}-\mathbf{w} \nabla \cdot \mathbf{u}+(\mathbf{w} \cdot \nabla) \mathbf{u}-(\mathbf{u} \cdot \nabla) \mathbf{w}$,

where $\mathbf{u}, \mathbf{w}$ are vector functions and $f$ is a scalar function, and since the flow is irrotational outside the vortex sheet, we may write

$$
\begin{aligned}
\nabla\left[\nabla \cdot\left(\rho_{c} \mathbf{u}_{c}\right)\right] & =\Delta\left(\rho_{c} \mathbf{u}_{c}\right)+\nabla \times\left[\nabla \times\left(\rho_{c} \mathbf{u}_{c}\right)\right] \\
& =\Delta\left(\rho_{c} \mathbf{u}_{c}\right)+\nabla \times\left(\nabla \rho_{c} \times \mathbf{u}_{c}\right) \\
& =\rho_{c} \Delta \mathbf{u}_{c}+2 \nabla \mathbf{u}_{c} \cdot \nabla \rho_{c}+\nabla \rho_{c} \nabla \cdot \mathbf{u}_{c} \\
& +\left(\mathbf{u}_{c} \cdot \nabla\right) \nabla \rho_{c}-\left(\nabla \rho_{c} \cdot \nabla\right) \mathbf{u}_{c} .
\end{aligned}
$$

By using the above identity in the equation (15), the nonlinear acoustic equation becomes

$$
\begin{aligned}
& \frac{\partial^{2} \mathbf{u}_{c}}{\partial t^{2}}+\frac{\partial}{\partial t}\left[\left(\mathbf{u}_{c} \cdot \nabla\right) \mathbf{u}_{c}\right]-c_{0}^{2} \Delta \mathbf{u}_{c} \\
&=-\frac{\nabla \rho_{c}}{\rho_{c}} \cdot \mathbf{u}_{c} \frac{\nabla p_{c}}{\rho_{c}}-\nabla \cdot \mathbf{u}_{c} \frac{\nabla p_{c}}{\rho_{c}} \\
&+2 c_{0}^{2} \nabla \mathbf{u}_{c} \cdot \frac{\nabla \rho_{c}}{\rho_{c}}+c_{0}^{2} \frac{\nabla \rho_{c}}{\rho_{c}} \nabla \cdot \mathbf{u}_{c} \\
&+c_{0}^{2} \frac{\left(\mathbf{u}_{c} \cdot \nabla\right) \nabla \rho_{c}}{\rho_{c}}-c_{0}^{2} \frac{\left(\nabla \rho_{c} \cdot \nabla\right) \mathbf{u}_{c}}{\rho_{c}} .
\end{aligned}
$$

Combining this with the state equation (13) we find that it is equivalent to

$$
\begin{aligned}
\frac{\partial^{2} \mathbf{u}_{c}}{\partial t^{2}}+\frac{\partial}{\partial t}\left[\left(\mathbf{u}_{c} \cdot \nabla\right) \mathbf{u}_{c}\right]-c_{0}^{2} \Delta \mathbf{u}_{c} \\
=-c_{0}^{2} \frac{\nabla \rho_{c}}{\rho_{c}} \cdot \mathbf{u}_{c} \frac{\nabla \rho_{c}}{\rho_{c}}+2 c_{0}^{2} \nabla \mathbf{u}_{c} \cdot \frac{\nabla \rho_{c}}{\rho_{c}} \\
+\frac{c_{0}^{2}}{\rho_{c}}\left[\left(\mathbf{u}_{c} \cdot \nabla\right) \nabla \rho_{c}-\left(\nabla \rho_{c} \cdot \nabla\right) \mathbf{u}_{c}\right] .
\end{aligned}
$$

Therefore, the motion inside the cavity may be described by the following equations

$$
\begin{aligned}
\frac{\partial^{2} \mathbf{u}_{c}}{\partial t^{2}}+\frac{\partial}{\partial t}[ & \left.\left(\mathbf{u}_{c} \cdot \nabla\right) \mathbf{u}_{c}\right]-c_{0}^{2} \Delta \mathbf{u}_{c} \\
= & -c_{0}^{2} \frac{\nabla \rho_{c}}{\rho_{c}} \cdot \mathbf{u}_{c} \frac{\nabla \rho_{c}}{\rho_{c}}+2 c_{0}^{2} \nabla \mathbf{u}_{c} \cdot \frac{\nabla \rho_{c}}{\rho_{c}} \\
& +\frac{c_{0}^{2}}{\rho_{c}}\left[\left(\mathbf{u}_{c} \cdot \nabla\right) \nabla \rho_{c}-\left(\nabla \rho_{c} \cdot \nabla\right) \mathbf{u}_{c}\right] \\
\frac{\partial \rho_{c}}{\partial t}= & -\nabla \cdot\left(\rho_{c} \mathbf{u}_{c}\right) \\
p_{c}= & c_{0}^{2} \rho_{c} .
\end{aligned}
$$

\section{Boundary Conditions}

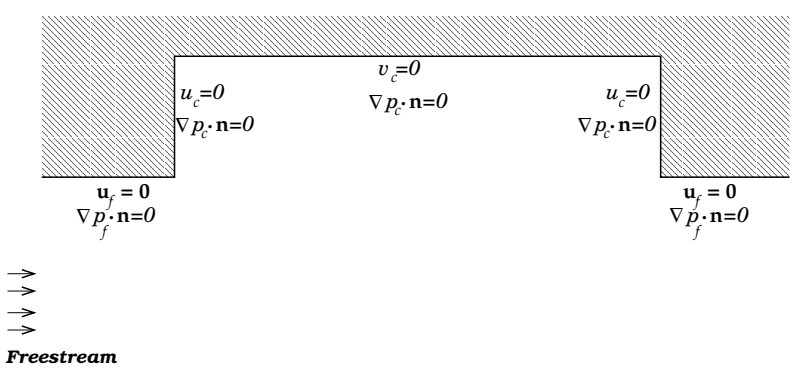

Figure 2: Boundary Conditions

We assume that the flow satisfies the nopenetration condition at the walls of the cavity and the no-slip condition at the walls outside the cavity, that is,

$$
\begin{aligned}
& \mathbf{u}_{c} \cdot \mathbf{n}=0, \quad \text { at cavity walls } \\
& \mathbf{u}_{f}=\mathbf{0}, \quad \text { at surfaces outside the cavity. }
\end{aligned}
$$

Here $\mathbf{n}$ denotes the unit vector normal to the wall (see Figure 2) and $\mathbf{0}$ is the null vector, that is, $\mathbf{0}=(0,0)$.

Also, we assume that there is no change in pressure magnitude in the normal direction at the walls, either inside or outside the cavity, i.e.

$$
\nabla p_{c} \cdot \mathbf{n}=0, \quad \nabla p_{f} \cdot \mathbf{n}=0
$$

at the walls.

Remark 4 The conditions on the pressure follow from the Navier Stokes equations (8) and equation (18) by considering the normal component to the walls of each term and assuming that the first and second derivatives of the velocity components are small and may be neglected.

\section{Interface Conditions}

The fluid is governed by different differential equations depending on whether it is above or below the shear layer. In this section we specify the interface or coupling conditions to be satisfied by the velocity and pressure at the shear layer. 


\section{Interface Condition for the Velocity}

As stated in the introduction, the shear layer generates and feeds acoustic waves inside the cavity which in turn, feed disturbances in the shear layer. This process induces different velocity distributions in the fluid above and below the shear layer, so that we have a fluid flowing one above the other having different velocity profiles, which is a special case of a Kelvin-Helmholtz instability. In particular, there is a discontinuity of the velocity in the tangential direction with respect to the shear layer, while the velocity in the normal direction remains continuous. (For more details concerning Kelvin-Helmholtz instabilities see [1], pp. 511-513; [5], pp. 15-17; [7], pp. $216 \mathrm{ss}$ ). Let us denote the velocity of the shear layer by $\mathbf{u}_{i s}$ and the unit vector normal to the shear layer by $\mathbf{n}$. Then, we have

$$
\mathbf{u}_{c} \cdot \mathbf{n}=\mathbf{u}_{i s} \cdot \mathbf{n}=\mathbf{u}_{f} \cdot \mathbf{n} .
$$

To develop appropriate interface conditions, the shear layer is viewed as an infinitesimally thin surface, a surface of discontinuity, separating the two fluid flows (see Figure 3).

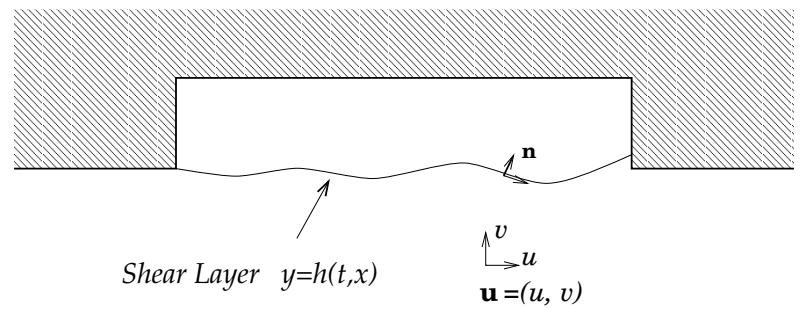

Figure 3: Interface Boundary

Remark 5 It is important to note that the tangential and normal components of the velocity with respect to the shear layer are not necessarily the horizontal and vertical components of the velocity. This is depicted in Figure 3.

We define $\mathcal{H}(t, x, y) \equiv h(t, x)-y$ where the shear layer interface is defined by $y_{i s}=h(t, x)$ and the $y$-axis is positive downward with $y=0$ at the mouth of the cavity. Then the normal to the shear layer at any time $t$ is given by

$$
\mathbf{n}=\frac{\nabla \mathcal{H}}{|\nabla \mathcal{H}|} .
$$

where

$$
\nabla \mathcal{H}=\left(\frac{\partial \mathcal{H}}{\partial x}, \frac{\partial \mathcal{H}}{\partial y}\right)=\left(\frac{\partial h}{\partial x},-1\right) .
$$

Thus the interface condition $\mathbf{u}_{c} \cdot \mathbf{n}=\mathbf{u}_{f} \cdot \mathbf{n}$ at $y=h$ becomes

$$
u_{c} \frac{\partial h}{\partial x}-v_{c}=u_{f} \frac{\partial h}{\partial x}-v_{f}
$$

or

$$
\left.\left(v_{f}-v_{c}\right)\right|_{y=h}=\left.\left(u_{f}-u_{c}\right)\right|_{y=h} \frac{\partial h}{\partial x} .
$$

Moreover, if we consider $u_{f}$ as equivalent to the freestream mean velocity $U$, the above identity may be approximated by

$$
\left.\left(v_{f}-v_{c}\right)\right|_{y=h}=\left(U-\left.u_{c}\right|_{y=h}\right) \frac{\partial h}{\partial x} .
$$

Remark 6 From the equation (23) we can see that $v_{f}=v_{c}$ if $h(t, x)$ is constant in $x$ (i.e., the shear layer is horizontal) or in the special case that the horizontal velocities $u_{f}$ and $u_{c}$ coincide (i.e., there is no shear layer).

Remark 7 In equation (24) we have approximated $u_{f}$ by the mean freestream velocity, $U$. We are, thus, assuming that the fluid flow below the shear layer is driven mainly by the freestream, and the perturbations produced by the shear layer are small compared to $U$.

Remark 8 Above the shear layer, the flow has zero horizontal mean velocity. However, at a particular position $u_{c}$ varies in direcction and magnitude, and its value may be high compared to its mean value 0. Approximating $u_{c}$ by its mean value would entail averaging the velocity in the whole cavity and for this reason we do not make this approximation on $u_{c}$.

\section{Interface Condition for the Pressure}

Another interfacial condition is given by the stress tensor, which must have a continuous normal component at the interface (see [1], [5], [7]). Since we neglect viscosity effects inside the cavity, the stress tensor inside the cavity is given by

$$
S_{c}=-p_{c} I,
$$

where $I$ denotes the identity operator. On the other hand, the stress tensor in the freestream flow is given by

$$
S_{f}=-p_{f} I+\mu\left(\nabla \mathbf{u}_{f}+\left(\nabla \mathbf{u}_{f}\right)^{T}\right),
$$


and thus, the stress condition reduces to

$$
-\left.p_{c} \mathbf{n}\right|_{y=h}=-\left.p_{f} \mathbf{n}\right|_{y=h}+\left.\mu \mathbf{n} \cdot\left(\nabla \mathbf{u}_{f}+\left(\nabla \mathbf{u}_{f}\right)^{T}\right)\right|_{y=h},
$$

or equivalently,

$$
\left.\left(p_{f}-p_{c}\right) \mathbf{n}\right|_{y=h}=\left.\mu \frac{\partial \mathbf{u}_{f}}{\partial \mathbf{n}}\right|_{y=h}+\left.\mu \mathbf{n} \cdot\left(\nabla \mathbf{u}_{f}\right)^{T}\right|_{y=h} .
$$

Furthermore, we assume that viscous terms of the stress in the direction normal to the shear layer are small compared to the pressure terms, i.e.

$\mu\left(\left.\frac{\partial \mathbf{u}_{f}}{\partial \mathbf{n}}\right|_{y=h}+\left.\mathbf{n} \cdot\left(\nabla \mathbf{u}_{f}\right)^{T}\right|_{y=h}\right)<<\left.\left(p_{f}-p_{c}\right) \mathbf{n}\right|_{y=h}$,

and therefore it may be neglected.

Remark 9 In other words, we are approximating the stress component normal to the shear layer by the pressure in the normal direction, i.e.

$$
\left.\mathbf{n} \cdot S_{f}\right|_{y=h} \approx-\left.p_{f} \mathbf{n}\right|_{y=h} .
$$

Hence, we obtain the continuity condition for the pressure at the interface

$$
\left.p_{f}\right|_{y=h}=\left.p_{c}\right|_{y=h} .
$$

Remark 10 If the fluid is considered inviscid everywhere, the condition of continuity for the pressure follows immediately from the continuity of the tensor.

\section{Approximation of the Interface Surface}

By considering the shear layer as the boundary where the interface conditions are imposed, we are dealing with a moving domain. For computational purposes we approximate the interface surface by the artificial boundary $y=0$, which is just the straight line connecting the ends of the cavity. Therefore, the interface conditions are further approximated by

$$
\begin{aligned}
& \left.v_{f}\right|_{y=0}=\left.v_{c}\right|_{y=0}+\left(U-\left.u_{c}\right|_{y=0}\right) \frac{\partial h}{\partial x} \\
& \left.p_{f}\right|_{y=0}=\left.p_{c}\right|_{y=0} .
\end{aligned}
$$

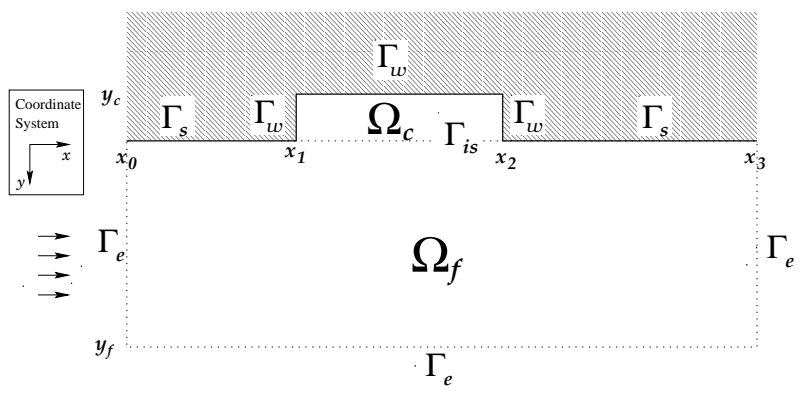

Figure 4: Domain

\section{THE FLOW-CAVITY MODEL}

We collect the equations, boundary conditions and interface conditions discussed in our proposed flow-cavity model.

Consider the open sets $\Omega_{f}, \Omega_{c}$ defined by

$$
\begin{gathered}
\Omega_{f}=\left\{(x, y): x \in\left(x_{0}, x_{3}\right), y \in\left(0, y_{f}\right)\right\}, \\
\Omega_{c}=\left\{(x, y): x \in\left(x_{1}, x_{2}\right), y \in\left(y_{c}, 0\right)\right\}
\end{gathered}
$$

with $x_{0}<<x_{1}<x_{2}<<x_{3}$ and $y_{c}<0<<y_{f}$ and boundaries $\partial \Omega_{f}=\Gamma_{f}$ and $\partial \Omega_{c}=\Gamma_{c}$.

Due to the different conditions on the boundaries, we subdivide $\Gamma_{f}$ and $\Gamma_{c}$ as follows

$$
\Gamma_{f}=\Gamma_{e} \cup \Gamma_{s} \cup \Gamma_{i s} \quad \Gamma_{c}=\Gamma_{w} \cup \Gamma_{i s}
$$

where $\Gamma_{e}, \Gamma_{s}, \Gamma_{i s}$ and $\Gamma_{w}$ are given by (see Figure 4)

$$
\begin{aligned}
\Gamma_{e}= & \left\{\left(x_{0}, y\right): y \in\left(0, y_{f}\right)\right\} \\
& \cup\left\{\left(x, y_{f}\right): x \in\left(x_{0}, x_{3}\right)\right\} \\
& \cup\left\{\left(x_{3}, y\right): y \in\left(0, y_{f}\right)\right\} \\
\Gamma_{s}= & \left\{(x, 0): x \in\left(x_{0}, x_{1}\right) \cup\left(x_{2}, x_{3}\right)\right\} \\
\Gamma_{i s}= & \left\{(x, 0): x \in\left(x_{1}, x_{2}\right)\right\} \\
\Gamma_{w}= & \left\{\left(x_{1}, y\right): y \in\left(y_{c}, 0\right)\right\} \\
& \cup\left\{\left(x, y_{c}\right): x \in\left(x_{1}, x_{2}\right)\right\} \\
& \cup\left\{\left(x_{2}, y\right): y \in\left(y_{c}, 0\right)\right\} .
\end{aligned}
$$

We consider the interaction flow-cavity within the domain $\Omega=\Omega_{f} \cup \Omega_{c}$. The flow motion can be described by combining the equations for the fluid in each subdomain, given by equations (8)-(10), (18)(20), boundary conditions for velocity and pressure, including (21) and (22), and the interface conditions for velocity and pressure, (27),(28). 
The model then consists of a set of coupled equations given by

$$
\begin{aligned}
& \frac{\partial \mathbf{u}_{f}}{\partial t}+\left(\mathbf{u}_{f} \cdot \nabla\right) \mathbf{u}_{f}=\frac{\mu}{\rho_{0}} \Delta \mathbf{u}_{f}-\frac{\nabla p_{f}}{\rho_{0}} \\
& \frac{\partial p_{f}}{\partial t}=-\nabla \cdot\left(p_{f} \mathbf{u}_{f}\right) \\
& p_{f}=c_{0}^{2} \rho_{f} \\
& \frac{\partial^{2} \mathbf{u}_{c}}{\partial t^{2}}-c_{0}^{2} \Delta \mathbf{u}_{c}=-\frac{\partial}{\partial t}\left[\left(\mathbf{u}_{c} \cdot \nabla\right) \mathbf{u}_{c}\right] \\
&- c_{0}^{2} \frac{\nabla \rho_{c}}{\rho_{c}} \cdot \mathbf{u}_{c} \frac{\nabla \rho_{c}}{\rho_{c}}+2 c_{0}^{2} \nabla \mathbf{u}_{c} \cdot \frac{\nabla \rho_{c}}{\rho_{c}} \\
&+\frac{c_{0}^{2}}{\rho_{c}}\left[\left(\mathbf{u}_{c} \cdot \nabla\right) \nabla \rho_{c}-\left(\nabla \rho_{c} \cdot \nabla\right) \mathbf{u}_{c}\right] \\
& \frac{\partial \rho_{c}}{\partial t}=-\nabla \cdot\left(\rho_{c} \mathbf{u}_{c}\right) \\
& p_{c}=c_{0}^{2} \rho_{c}
\end{aligned}
$$

where $\mathbf{u}_{f}, p_{f}, \rho_{f}$ are defined on $\Omega_{f}$ and $\mathbf{u}_{c}, p_{c}, \rho_{c}$ are defined on $\Omega_{c}$, with boundary conditions

$$
\begin{aligned}
& u_{f} / c=M, \quad v_{f}=0 \quad p_{f}=p_{e} \quad \text { on } \Gamma_{e} \\
& \mathbf{u}_{f}=\mathbf{0}, \quad \nabla p_{f} \cdot \mathbf{n}=0 \quad \text { on } \Gamma_{s} \\
& \mathbf{u}_{c} \cdot \mathbf{n}=0, \quad \nabla p_{c} \cdot \mathbf{n}=0 \quad \text { on } \Gamma_{w}
\end{aligned}
$$

where $p_{e}$ is a given pressure distribution on $\Gamma_{e}$, and coupling conditions on $\Gamma_{i s}$

$$
\begin{aligned}
& \left.v_{f}\right|_{y=0}=\left.v_{c}\right|_{y=0}+\left(U-\left.u_{c}\right|_{y=0}\right) \frac{\partial h}{\partial x}, \\
& \left.p_{f}\right|_{y=0}=\left.p_{c}\right|_{y=0} .
\end{aligned}
$$

Here we have assumed that the artificial boundary, denoted by $\Gamma_{e}$, is far enough from the cavity so that there are no effects from fluid/structure interaction.

\section{WEAK FORM}

For both theoretical and computational considerations it is often convenient to write a model such as that derived above in a weak or variational form. We do that here, following the general ideas used in [2] and [10].
First, we define the framework for the fluid/structure interaction model given above. Let $V_{f}$ be the subspace of $\left[H^{1}\left(\Omega_{f}\right)\right]^{2}$ defined by

$$
\begin{aligned}
V_{f}=\left\{\Psi \in\left[H^{1}\left(\Omega_{f}\right)\right]^{2}:\right. & \nabla \cdot \Psi=0 \text { in } \Omega_{f}, \\
& \left.\Psi=\mathbf{0} \text { on } \Gamma_{f}-\Gamma_{i s}\right\},
\end{aligned}
$$

equipped with the $\left[H^{1}\left(\Omega_{f}\right)\right]^{2}$-norm

$$
\|\mathbf{u}\|_{V_{f}}^{2}=\|\mathbf{u}\|_{\left[H^{1}\left(\Omega_{f}\right)\right]^{2}}^{2}=\sum_{i=1}^{2}\left\|u_{i}\right\|_{H^{1}\left(\Omega_{f}\right)}^{2}
$$

and

$$
|\mathbf{u}|_{V_{f}}^{2}=\sum_{i=1}^{2}\left|\nabla u_{i}\right|_{\left[L^{2}\left(\Omega_{f}\right)\right]^{2}}^{2} .
$$

Let $W_{f}$ be the subspace of $H^{1}\left(\Omega_{f}\right)$ defined by

$$
W_{f}=\left\{\phi \in H^{1}\left(\Omega_{f}\right): \phi=0 \text { on } \Gamma_{e}\right\}
$$

equipped with the $H^{1}\left(\Omega_{f}\right)$-norm, and let $V_{c}$ be the subspace of $\left[H^{1}\left(\Omega_{c}\right)\right]^{2}$ defined by

$$
V_{c}=\left\{\bar{\Psi} \in\left[H^{1}\left(\Omega_{c}\right)\right]^{2}: \bar{\Psi} \cdot \mathbf{n}=0 \text { on } \Gamma_{w}\right\},
$$

equipped with the $\left[H^{1}\left(\Omega_{c}\right)\right]^{2}$-norm.

Let $\Psi \in V_{f}, \phi \in W_{f}, \bar{\Psi} \in V_{c}$ and $\bar{\phi} \in H^{1}\left(\Omega_{c}\right)$ and consider the variational form of the system (29)(34) with $\mathbf{u}_{f} \in V_{f}, \quad p_{f}$ and $\rho_{f} \in W_{f}, \quad \mathbf{u}_{c} \in V_{c}$ and $p_{c}, \rho_{c} \in H^{2}\left(\Omega_{c}\right)$.

We will seek solutions that satisfy homogeneous boundary conditions since these can be translated into solutions satisfying (35) in a straight forward manner (see [2]).

We then have, along with the coupling equations (38), the system

$$
\begin{gathered}
\left(\frac{\partial \mathbf{u}_{f}}{\partial t}, \Psi\right)+\left(\left(\mathbf{u}_{f} \cdot \nabla\right) \mathbf{u}_{f}, \Psi\right)=\left(\frac{1}{\rho_{0}} \nabla \cdot \tilde{S}, \Psi\right) \\
\left(\frac{\partial p_{f}}{\partial t}, \phi\right)+\left(\nabla \cdot\left(p_{f} \mathbf{u}_{f}\right), \phi\right)=0 \\
\left(p_{f}, \phi\right)-c_{0}^{2}\left(\rho_{f}, \phi\right)=0 \\
\left(\frac{\partial^{2} \mathbf{u}_{c}}{\partial t^{2}}, \bar{\Psi}\right)+\left(\frac{\partial}{\partial t}\left[\left(\mathbf{u}_{c} \cdot \nabla\right) \mathbf{u}_{c}\right], \bar{\Psi}\right)
\end{gathered}
$$




$$
\begin{aligned}
& -c_{0}^{2}\left(\Delta \mathbf{u}_{c}, \bar{\Psi}\right)+\left(c_{0}^{2} \frac{\nabla \rho_{c}}{\rho_{c}} \cdot \mathbf{u}_{c} \frac{\nabla \rho_{c}}{\rho_{c}}, \bar{\Psi}\right) \\
& -\left(\frac{c_{0}^{2}}{\rho_{c}}\left[\left(\mathbf{u}_{c} \cdot \nabla\right) \nabla \rho_{c}-\left(\nabla \rho_{c} \cdot \nabla\right) \mathbf{u}_{c}\right], \bar{\Psi}\right) \\
& -2 c_{0}^{2}\left(\nabla \mathbf{u}_{c} \cdot \frac{\nabla \rho_{c}}{\rho_{c}}, \bar{\Psi}\right)=0 \\
& \left(\frac{\partial \rho_{c}}{\partial t}, \bar{\phi}\right)+\left(\nabla \cdot\left(\rho_{c} \mathbf{u}_{c}\right), \bar{\phi}\right)=0 \\
& \left(p_{c}, \bar{\phi}\right)-c_{0}^{2}\left(\rho_{c}, \bar{\phi}\right)=0,
\end{aligned}
$$

where $\tilde{S}$ is defined by

$$
\tilde{S} \equiv-p_{f} I+\mu \nabla \mathbf{u}_{f} .
$$

Remark 11 Recall that for $\mu$ constant the divergence of the stress tensor, given by

$$
\nabla \cdot S_{f}=\nabla \cdot\left\{-p_{f} I+\mu\left[\nabla \mathbf{u}_{f}+\left(\mathbf{u}_{f}\right)^{T}\right]\right\},
$$

reduces to

$$
\nabla \cdot S_{f}=\nabla \cdot\left(-p_{f} I+\mu \nabla \mathbf{u}_{f}\right) .
$$

(see Remark 1). Thus, we have

$$
\nabla \cdot S_{f}=\nabla \cdot \tilde{S}
$$

We note that

$$
((\mathbf{u} \cdot \nabla) \mathbf{v}, \mathbf{w})=-\left(\mathbf{v} \cdot(\nabla \mathbf{w})^{T}, \mathbf{u}\right)+(\mathbf{v} \mathbf{n} \cdot \mathbf{u}, \mathbf{w})_{\Gamma_{i s}}
$$

for $\mathbf{u}, \mathbf{v}, \mathbf{w} \in V_{f}$. This identity follows from

$$
\nabla \cdot(v \mathbf{u})=(\mathbf{u} \cdot \nabla) v+v \nabla \cdot \mathbf{u},
$$

for $v \in H^{1}\left(\Omega_{f}\right), \mathbf{u} \in\left[H^{1}\left(\Omega_{f}\right)\right]^{2}$, combined with Green's formula

$$
(\nabla \cdot \Psi, \phi)_{\Omega_{f}}=(\mathbf{n} \cdot \Psi, \phi)_{\partial \Omega_{f}}-(\Psi, \nabla \phi)_{\Omega_{f}},
$$

where $\Psi \in\left[H^{1}\left(\Omega_{f}\right)\right]^{2}, \phi \in H^{1}\left(\Omega_{f}\right)$. The arguments for (45) are given by

$$
\begin{aligned}
((\mathbf{u} \cdot \nabla) \mathbf{v}, \mathbf{w})= & \left((\mathbf{u} \cdot \nabla) v_{1}, w_{1}\right)+\left((\mathbf{u} \cdot \nabla) v_{2}, w_{2}\right) \\
= & \left(\nabla \cdot\left(v_{1} \mathbf{u}\right)-v_{1} \nabla \cdot \mathbf{u}, w_{1}\right) \\
& +\left(\nabla \cdot\left(v_{2} \mathbf{u}\right)-v_{2} \nabla \cdot \mathbf{u}, w_{2}\right) \\
= & -\left(v_{1} \mathbf{u}, \nabla w_{1}\right)+\left(v_{1} \mathbf{n} \cdot \mathbf{u}, w_{1}\right)_{\Gamma_{f}} \\
& -\left(v_{2} \mathbf{u}, \nabla w_{2}\right)+\left(v_{2} \mathbf{n} \cdot \mathbf{u}, w_{2}\right)_{\Gamma_{f}} \\
= & -\left(\mathbf{v} \cdot(\nabla \mathbf{w})^{T}, \mathbf{u}\right)+(\mathbf{v n} \cdot \mathbf{u}, \mathbf{w})_{\Gamma_{i s}},
\end{aligned}
$$

where we recall that $\nabla \cdot \mathbf{u}=0$ in $\Omega_{f}$ and $\mathbf{w}=\mathbf{0}$ on $\Gamma_{f}-\Gamma_{i s}$.

Again using Green's formula and introducing the coefficient of kinematic viscosity denoted by $\nu=$ $\mu / \rho_{0}$, we see that the equation (39) becomes

$$
\begin{aligned}
& \left(\frac{\partial \mathbf{u}_{f}}{\partial t}, \Psi\right)-\left(\mathbf{u}_{f} \cdot(\nabla \Psi)^{T}, \mathbf{u}_{f}\right)-\left(\mathbf{u}_{f} v_{f}, \Psi\right)_{\Gamma_{i s}} \\
& \quad+\left(\nu \nabla \mathbf{u}_{f}, \nabla \Psi\right)+\left(p_{f} / \rho_{0} \mathbf{n}, \Psi\right)_{\Gamma_{i s}}=0 .(48)
\end{aligned}
$$

Remark 12 Note that in the above computation we assumed

$$
\frac{\partial \mathbf{u}_{f}}{\partial \mathbf{n}}=\nabla \mathbf{u}_{f} \cdot \mathbf{n} \approx 0 \quad \text { on } \Gamma_{i s},
$$

so that

$$
\left(\mathbf{n} \cdot \tilde{S} / \rho_{0}, \Psi\right)_{\Gamma_{i s}} \approx-\left(\mathbf{n} p_{f} / \rho_{0}, \Psi\right)_{\Gamma_{i s}} .
$$

Remark 13 In the equation (48) we have used the fact that the outward normal vector to $\Gamma_{\text {is }}$ is pointing upward, away form $\Omega_{f}$, and because of the coordinate system chosen, we have

$$
\mathbf{u}_{f} \cdot \mathbf{n}=-v_{f} .
$$

Consider now the equation (42) given by

$$
\begin{aligned}
\left(\frac{\partial^{2} \mathbf{u}_{c}}{\partial t^{2}}, \bar{\Psi}\right)+\left(\frac{\partial}{\partial t}\left[\left(\mathbf{u}_{c} \cdot \nabla\right) \mathbf{u}_{c}\right], \bar{\Psi}\right) & \\
& -c_{0}^{2}\left(\Delta \mathbf{u}_{c}, \bar{\Psi}\right)+\left(c_{0}^{2} \frac{\nabla \rho_{c}}{\rho_{c}} \cdot \mathbf{u}_{c} \frac{\nabla \rho_{c}}{\rho_{c}}, \bar{\Psi}\right) \\
& -\left(\frac{c_{0}^{2}}{\rho_{c}}\left[\left(\mathbf{u}_{c} \cdot \nabla\right) \nabla \rho_{c}-\left(\nabla \rho_{c} \cdot \nabla\right) \mathbf{u}_{c}\right], \bar{\Psi}\right) \\
& -2 c_{0}^{2}\left(\nabla \mathbf{u}_{c} \cdot \frac{\nabla \rho_{c}}{\rho_{c}}, \bar{\Psi}\right)=0 .
\end{aligned}
$$

The second term on the left side can be written as

$$
\begin{aligned}
\left(\frac{\partial}{\partial t}\left[\left(\mathbf{u}_{c} \cdot \nabla\right) \mathbf{u}_{c}\right], \bar{\Psi}\right)= & \left(\left(\frac{\partial \mathbf{u}_{c}}{\partial t} \cdot \nabla\right) \mathbf{u}_{c}, \bar{\Psi}\right) \\
+ & \left(\left(\mathbf{u}_{c} \cdot \nabla\right) \frac{\partial \mathbf{u}_{c}}{\partial t}, \bar{\Psi}\right) \cdot(
\end{aligned}
$$

By arguments similar to those below equation (45), we find the following identity for $\mathbf{u}, \mathbf{v}, \mathbf{w} \in V_{c}$ :

$$
\begin{aligned}
((\mathbf{u} \cdot \nabla) \mathbf{v}, \mathbf{w})= & -\left(\mathbf{v} \cdot(\nabla \mathbf{w})^{T}, \mathbf{u}\right)-(\mathbf{v} \nabla \cdot \mathbf{u}, \mathbf{w}) \\
& +(\mathbf{v} \mathbf{n} \cdot \mathbf{u}, \mathbf{w})_{\Gamma_{i s}} .
\end{aligned}
$$


Applying this identity to each term on the right side of (49), we find

$$
\begin{aligned}
\left(\frac{\partial}{\partial t}\right. & {\left.\left[\left(\mathbf{u}_{c} \cdot \nabla\right) \mathbf{u}_{c}\right], \bar{\Psi}\right) } \\
= & -\left(\mathbf{u}_{c} \cdot(\nabla \bar{\Psi})^{T}, \frac{\partial \mathbf{u}_{c}}{\partial t}\right)-\left(\mathbf{u}_{c} \nabla \cdot\left(\frac{\partial \mathbf{u}_{c}}{\partial t}\right), \bar{\Psi}\right) \\
& +\left(\mathbf{u}_{c} \mathbf{n} \cdot \frac{\partial \mathbf{u}_{c}}{\partial t}, \bar{\Psi}\right)_{\Gamma_{i s}}-\left(\frac{\partial \mathbf{u}_{c}}{\partial t} \cdot(\nabla \bar{\Psi})^{T}, \mathbf{u}_{c}\right) \\
& -\left(\frac{\partial \mathbf{u}_{c}}{\partial t} \nabla \cdot \mathbf{u}_{c}, \bar{\Psi}\right)+\left(\frac{\partial \mathbf{u}_{c}}{\partial t} \mathbf{n} \cdot \mathbf{u}_{c}, \bar{\Psi}\right)_{\Gamma_{i s}} \\
= & -\left(\mathbf{u}_{c} \cdot(\nabla \bar{\Psi})^{T}, \frac{\partial \mathbf{u}_{c}}{\partial t}\right)-\left(\frac{\partial \mathbf{u}_{c}}{\partial t} \cdot(\nabla \bar{\Psi})^{T}, \mathbf{u}_{c}\right) \\
& -\left(\frac{\partial}{\partial t}\left(\mathbf{u}_{c} \nabla \cdot \mathbf{u}_{c}\right), \bar{\Psi}\right)+\left(\frac{\partial}{\partial t}\left(\mathbf{u}_{c} \mathbf{n} \cdot \mathbf{u}_{c}\right), \bar{\Psi}\right)_{\Gamma_{i s}} .
\end{aligned}
$$

We substitute this back into equation (42) to obtain

$$
\begin{aligned}
\left(\frac{\partial^{2} \mathbf{u}_{c}}{\partial t^{2}}, \bar{\Psi}\right)+c_{0}^{2}\left(\nabla \mathbf{u}_{c}, \nabla \bar{\Psi}\right)+c_{0}^{2}\left(\mathbf{n} \cdot \nabla \mathbf{u}_{c}, \bar{\Psi}\right)_{\Gamma_{c}} \\
\quad-\left(\mathbf{u}_{c} \cdot(\nabla \bar{\Psi})^{T}, \frac{\partial \mathbf{u}_{c}}{\partial t}\right)-\left(\frac{\partial \mathbf{u}_{c}}{\partial t} \cdot(\nabla \bar{\Psi})^{T}, \mathbf{u}_{c}\right) \\
\quad-\left(\frac{\partial}{\partial t}\left(\mathbf{u}_{c} \nabla \cdot \mathbf{u}_{c}\right), \bar{\Psi}\right)+\left(\frac{\partial}{\partial t}\left(\mathbf{u}_{c} v_{c}\right), \Psi\right)_{\Gamma_{i s}} \\
\quad+\left(c_{0}^{2} \frac{\nabla \rho_{c}}{\rho_{c}} \cdot \mathbf{u}_{c} \frac{\nabla \rho_{c}}{\rho_{c}}, \bar{\Psi}\right)-2 c_{0}^{2}\left(\nabla \mathbf{u}_{c} \cdot \frac{\nabla \rho_{c}}{\rho_{c}}, \bar{\Psi}\right) \\
-\left(\frac{c_{0}^{2}}{\rho_{c}}\left[\left(\mathbf{u}_{c} \cdot \nabla\right) \nabla \rho_{c}-\left(\nabla \rho_{c} \cdot \nabla\right) \mathbf{u}_{c}\right], \bar{\Psi}\right) \\
\quad=0 .
\end{aligned}
$$

Therefore, by using equations (48) and (51) and (38), the variational formulation for the system (29)(34) may be written as the coupling equation (38) along with the equations

$$
\begin{aligned}
& \left(\frac{\partial \mathbf{u}_{f}}{\partial t}, \Psi\right)+a\left(\mathbf{u}_{f}, \Psi\right)-b_{1}\left(\mathbf{u}_{f}, \Psi, \mathbf{u}_{f}\right) \\
& -\left(\mathbf{u}_{f} v_{f}, \Psi\right)_{\Gamma_{i s}}+\left(p_{f} / \rho_{0} \mathbf{n}, \Psi\right)_{\Gamma_{i s}}=0 \\
& \left(\frac{\partial p_{f}}{\partial t}, \phi\right)-e\left(\mathbf{u}_{f}, p_{f}, \phi\right)-\left(p_{f} v_{f}, \phi\right)_{\Gamma_{i s}}=0(53) \\
& \left(p_{f}-c_{0}^{2} \rho_{f}, \phi\right)=0 \\
& \left(\frac{\partial^{2} \mathbf{u}_{c}}{\partial t^{2}}, \bar{\Psi}\right)+c_{0}^{2} \bar{a}\left(\mathbf{u}_{c}, \bar{\Psi}\right)
\end{aligned}
$$

$$
\begin{aligned}
& -\left[\bar{b}_{1}\left(\mathbf{u}_{c}, \bar{\Psi}, \frac{\partial \mathbf{u}_{c}}{\partial t}\right)+\bar{b}_{1}\left(\frac{\partial \mathbf{u}_{c}}{\partial t}, \bar{\Psi}, \mathbf{u}_{c}\right)\right] \\
& -\left[\bar{b}_{2}\left(\frac{\partial \mathbf{u}_{c}}{\partial t}, \mathbf{u}_{c}, \bar{\Psi}\right)+\bar{b}_{2}\left(\mathbf{u}_{c}, \frac{\partial \mathbf{u}_{c}}{\partial t}, \bar{\Psi}\right)\right] \\
& +c_{0}^{2} \bar{d}\left(\mathbf{u}_{c}, \frac{\nabla \rho_{c}}{\rho_{c}}, \bar{\Psi}\right)-2 c_{0}^{2} \bar{b}_{11}\left(\mathbf{u}_{c}, \frac{\nabla \rho_{c}}{\rho_{c}}, \bar{\Psi}\right) \\
& -c_{0}^{2}\left[\bar{b}_{3}\left(\mathbf{u}_{c}, \frac{\nabla \rho_{c}}{\rho_{c}}, \bar{\Psi}\right)-\bar{b}_{3}\left(\frac{\nabla \rho_{c}}{\rho_{c}}, \mathbf{u}_{c}, \bar{\Psi}\right)\right] \\
& +c_{0}^{2}\left(\mathbf{n} \cdot \nabla \mathbf{u}_{c}, \bar{\Psi}\right)_{\Gamma_{c}}+\left(\frac{\partial}{\partial t}\left(\mathbf{u}_{c} v_{c}\right), \Psi\right)_{\Gamma_{i s}}=0 \\
& \left(\frac{\partial \rho_{c}}{\partial t}, \bar{\phi}\right)-\bar{e}\left(\mathbf{u}_{c}, \rho_{c}, \bar{\phi}\right)+\left(v_{c} \rho_{c}, \bar{\phi}\right)_{\Gamma_{i s}}=0 \\
& \left(p_{c}-c_{0}^{2} \rho_{c}, \bar{\phi}\right)=0,
\end{aligned}
$$

where the forms $a(\cdot, \cdot), b_{1}(\cdot, \cdot, \cdot), e(\cdot, \cdot), \quad \bar{a}(\cdot, \cdot)$, $\bar{b}_{1}(\cdot, \cdot, \cdot), \bar{b}_{11}(\cdot, \cdot, \cdot), \bar{b}_{2}(\cdot, \cdot, \cdot), \bar{b}_{3}(\cdot, \cdot, \cdot), \bar{d}(\cdot, \cdot)$ and $\bar{e}(\cdot, \cdot, \cdot)$ are defined as follows

$$
\begin{aligned}
& a(\mathbf{u}, \mathbf{v})=(\nu \nabla \mathbf{u}, \nabla \mathbf{v}), \\
& b_{1}(\mathbf{u}, \mathbf{v}, \mathbf{w})=\left(\mathbf{u} \cdot(\nabla \mathbf{v})^{T}, \mathbf{w}\right), \\
& e(\mathbf{u}, p, q)=(p \mathbf{u}, \nabla q), \\
& \bar{a}(\overline{\mathbf{u}}, \overline{\mathbf{v}})=(\nabla \overline{\mathbf{u}}, \nabla \overline{\mathbf{v}}), \\
& \bar{b}_{1}(\overline{\mathbf{u}}, \overline{\mathbf{v}}, \overline{\mathbf{w}})=\left(\overline{\mathbf{u}} \cdot(\nabla \overline{\mathbf{v}})^{T}, \overline{\mathbf{w}}\right), \\
& \bar{b}_{11}(\overline{\mathbf{u}}, \overline{\mathbf{v}}, \overline{\mathbf{w}})=(\nabla \overline{\mathbf{u}} \cdot \overline{\mathbf{v}}, \overline{\mathbf{w}}), \\
& \bar{b}_{2}(\overline{\mathbf{u}}, \overline{\mathbf{v}}, \overline{\mathbf{w}})=(\overline{\mathbf{u}} \nabla \cdot \overline{\mathbf{v}}, \overline{\mathbf{w}}), \\
& \bar{b}_{3}(\overline{\mathbf{u}}, \overline{\mathbf{v}}, \overline{\mathbf{w}})=((\overline{\mathbf{u}} \cdot \nabla) \overline{\mathbf{v}}, \overline{\mathbf{w}}), \\
& \bar{d}(\overline{\mathbf{u}}, \overline{\mathbf{v}}, \overline{\mathbf{w}})=((\overline{\mathbf{u}} \cdot \overline{\mathbf{v}}) \overline{\mathbf{v}}, \overline{\mathbf{w}}), \\
& \bar{e}(\overline{\mathbf{u}}, \bar{r}, \bar{q})=(\bar{r} \mathbf{u}, \nabla \bar{q}),
\end{aligned}
$$

with $\mathbf{u}, \mathbf{v}, \mathbf{w} \in V_{f}, p, q \in W_{f}, \overline{\mathbf{u}}, \overline{\mathbf{v}}, \overline{\mathbf{w}} \in V_{c}$ and $\bar{r}, \bar{q} \in$ $H^{1}\left(\Omega_{c}\right)$.

\section{REFERENCES}

[1] G.K. Batchelor, An Introduction to Fluid Dynamics, Cambridge University Press, 1967.

[2] H.T. Banks and K. Ito, "Structural Actuator Control of Fluid/Structure Interactions," Proc. $33^{\text {rd }}$ Conference on Decision and Control, Lake Buena Vista, Fl, Dec. 1994, pp.283-288.

[3] A.B. Cain, W.W. Bower, F.McCotter and W.W.Romer, "Modeling and Prediction of Weapons Bay Acoustic Amplitude and Frequency," P.O. P61100-5016, Technical Report Submitted to VEDA Inc., Feb. 1996. 
[4] A.J. Chorin and J.E. Marsden, A Mathematical Introduction to Fluid Mechanics, SpringerVerlag, NY, 1990.

[5] P.G. Drazin and W.H. Reid, Hydrodynamic Stability, Cambridge University Press, 1981.

[6] H.H. Heller and D.B. Bliss, "Flow-Induced Pressure Fluctuations in Cavities and Concepts for their Suppression," AIAA $2^{\text {nd }}$ Aero-Acoustics Conference, Hampton, VA, March 1975, Paper 75-491.

[7] D.Y. Hsieh and S.P. Ho, Wave and Stability in Fluids, World Scientific Pub., Singapore, 1994.

[8] D. Rockwell and E. Naudascher, "ReviewSelf-Sustaining Oscillations of Flow Past Cavities," Journal of Fluids Engineering, Vol. 100, June 1978, pp.152-165.

[9] R.L. Sarno and M.E. Franke, "Suppression of Flow-Induced Pressure Oscillations in Cavities," Journal of Aircraft, Vol. 31, No. 1, Jan.-Feb. 1994, pp.90-96.

[10] R. Temam, Navier Stokes Equations: Theory and Numerical Analysis, North-Holland, Amsterdam, 1971.

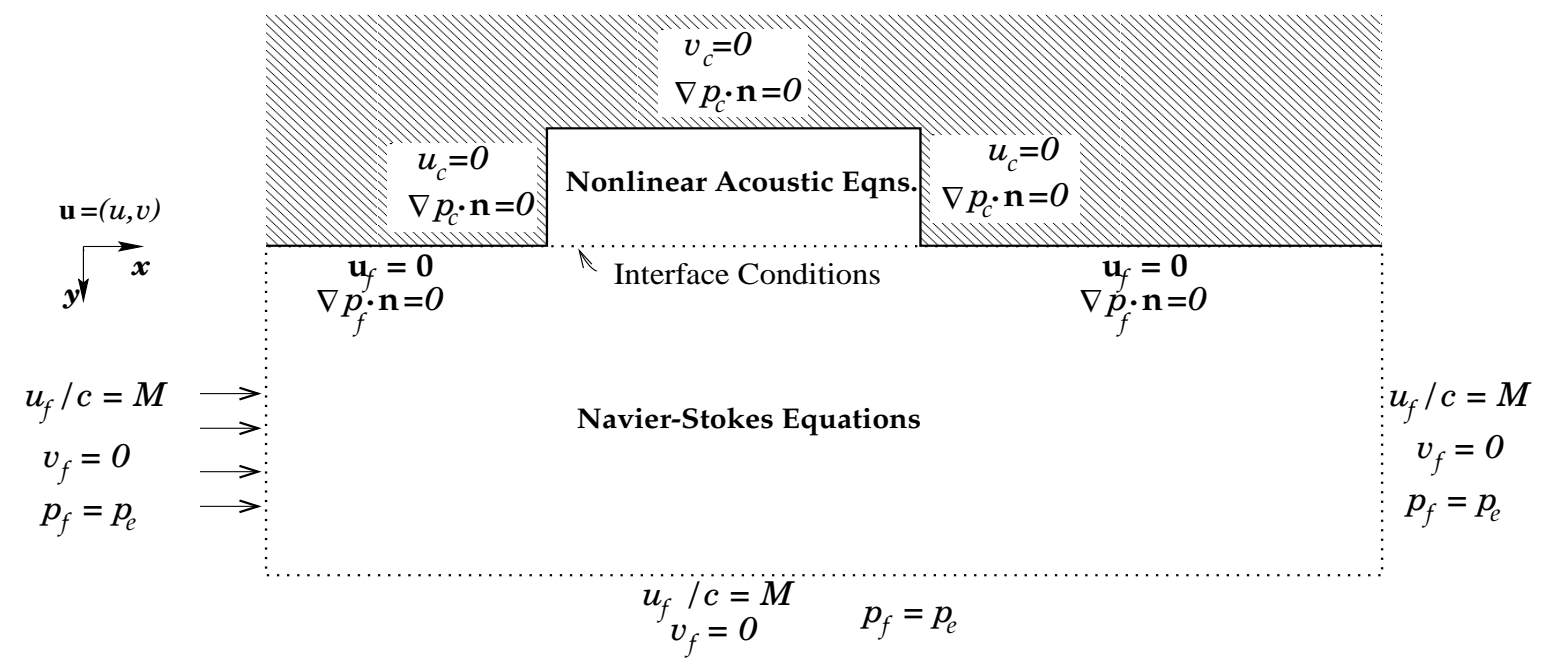

Figure 5: Flow-Cavity Model 